\title{
Dielectric and mechanical relaxation in PMPS, BMC and their mixtures
}

\author{
P.G. Santangelo ${ }^{a, *}$, C.M. Roland ${ }^{a}$, K.L. Ngai ${ }^{a}$, A.K. Rizos ${ }^{b}$, H. Katerinopoulos ${ }^{b}$ \\ a Naval Research Laboratory, Washington, DC 20375-5342, USA \\ ${ }^{b}$ Foundation for Research and Technology-Hellas and University of Crete, Department of Chemistry, Heraklion 71409 , \\ Crete, Greece
}

\begin{abstract}
Dielectric and dynamical mechanical measurements have been performed on mixtures of the small molecule glassforming liquid 1,1-bis(p-methoxyphenyl) cyclohexane (BMC) with poly(methylphenylsiloxane) (PMPS). Earlier measurements had indicated that the BMC dynamics are modified in a very interesting way by the presence of the polymer, despite the equivalence of their glass transition temperatures, $T_{\mathrm{g}}$. By varying the molecular weight of the polymer (and hence $T_{\mathrm{g}}$, the BMC mobility is anomalously enhanced in mixtures of high $T_{\mathrm{g}}$ PMPS. An interpretation for these results based on the coupling model of relaxation is suggested.
\end{abstract}

\section{Introduction}

Although the detailed consequences of mixing on the local dynamics are not fully understood [1], the respective relaxation times measured for a polymer and a small molecule glass former are expected to be closer in magnitude when mixed than in their respective pure states. A free-volume concept of segmental relaxation, whereby the glass transition temperature, $T_{\mathrm{g}}$, influences local dynamics through the monomeric friction coefficient, suggests that a polymer-rich mixture containing a lower $T_{\mathrm{g}}$ diluent will exhibit a decreased relaxation time over

* Corresponding author. Tel: +1-202404 7352. Telefax: +12027670546. that of the neat polymer (i.e. plasticization). That is, if the diluent dynamics are faster than the polymer dynamics, the segmental relaxation of the polymer will be enhanced by addition of the small molecule. In the less frequent case of the small molecule diluent having a higher $T_{\mathrm{g}}$, the segmental relaxation time of the polymer will be increased with addition of the diluent (i.e., anti-plasticization).

Further, it has become evident that the solvent rotational mobility and local friction coefficient can be significantly altered with the addition of small amounts of polymer [2-4]. In a solvent-rich mixture, it is expected that addition of a higher $T_{\mathrm{g}}$ polymer will slow the reorientational relaxation time of the solvent, while the addition of a lower $T_{\mathrm{g}}$ polymer will decrease the solvent relaxation time.

These behaviors have been observed for various mixtures having components with very dissimilar 
glass transition temperatures. The glass transition temperature of the mixture as well as the relaxation times of each component fall between those of the neat materials. More interesting situations can arise when the glass transition temperatures of the neat components are nearly equal.

One notable example is the anomaly observed in polychlorinated biphenyl (PCB, trade-named Aroclor) containing a small quantity of poly(vinylethylene) (PVE). The reorientational relaxation time of the PCB was reduced by addition of the polymer, which is remarkable considering that the $T_{\mathrm{g}}$ of the PVE was approximately $19 \mathrm{~K}$ above that of the neat PCB $[5,6]$.

An interpretation of this phenomenon was presented [5] based on the coupling model of relaxation $[7,8]$. According to the coupling model, the observed relaxation time, $\tau^{*}$, depends both on the local friction and on the extent to which nonbonded neighboring molecules constrain the local motion; thus,

$\tau^{*}=\left[(1-n) \omega_{\mathrm{c}}^{n} \tau_{0}\right]^{1 /(1-n)}$,

where $\omega_{c}^{-1}$ defines the time for inception of intermolecular constraints and $n$ is a measure of the severity of intermolecular constraints. The local friction factor is reflected in the magnitude of $\tau_{0}$, the relaxation time in the absence of intermolecular constraints. According to the coupling model, PCB is weakly intermolecularly coupled (small $n$ ), in contrast to PVE, whose highly cooperative segmental relaxation has been ascribed to the presence of the sterically hindering pendant vinyl groups [9-11]. Even though the PVE has a higher $T_{\mathrm{g}}$ than PCB, the non-linearity of Eq. (1) suggests that the polymer may have a smaller friction coefficient (i.e., smaller $\left.\tau_{0}\right)$. The intermolecular coupling of the polymer is alleviated by dilution with a smaller $n$ effecting smaller $\tau^{*}$ (Eq. (1)). Its relaxation time could actually become shorter than that of the PCB. This is in fact predicted from Eq. (1), where the relaxation time in the absence of intermolecular constraints is calculated to be less for the PVE than for the PCB, $\tau_{0, \mathrm{PVE}}<\tau_{0, \mathrm{PCB}}$ [5]. Hence, the coupling model predicts a reversal in relaxation times since $\tau_{\mathrm{PVE}}^{*}>\tau_{\mathrm{PCB}}^{*}$. This implies that, as observed, addition of polymer would speed up relaxation of the PCB. In their application of the coupling model, Rizos and Ngai [5] gave no consideration to possible modification of $\tau_{0}$ s due to a non-zero excess volume of mixing (i.e., a non-ideal mixing volume [12-14]. Actually, recent results with higher molecular weight and greater vinyl content PVE reveal another source of the anomalous behavior in this system, originating from an excess mixing volume that is atypically positive [6].

In general then, we can expect that observed changes in relaxation times upon mixing may not parallel the respective glass transition temperatures of the pure components, particularly when the latter are close. This is due to the fact that the local friction coefficient is governed by the magnitude of $\tau_{0}$, rather than $\tau^{*}$ (or the temperature at which $\tau^{*}$ assumes the value defining $T_{\mathrm{g}}$ ). Of course, only the latter is actually measured for the neat components.

In a recent tracer diffusion study of photochromic dye molecules in polymer mixtures of 20000 molecular weight poly(methylphenylsiloxane) (PMPS) with 1,1-bis(p-methoxyphenyl) cyclohexone (BMC), the temperature dependence of the translational diffusion coefficient, $D$, of the photochromic molecules in solvent-rich BMC/PMPS mixtures was found to have significantly smaller values than those predicted by theory [15]. These results are extremely interesting considering that both the polymer and small molecule had equivalent glass transition temperatures. Using photochromic probe molecules of the same size as the BMC, the results indicate that the segmental dynamics of PMPS are strongly modified by the presence of $\mathrm{BMC}$, notwithstanding the equivalence of their glass transition temperatures.

Stimulated by these results, dynamic mechanical measurements in the vicinity of the glass transition temperature were carried out in a recent study on BMC, PMPS of different molecular weights (and thus different $T_{\mathrm{g}} \mathrm{s}$ ), and on both polymer and BMC-rich mixtures [16]. In this previous study [16], coupling model-derived arguments were advanced to explain the anomalous solvent modification in mixtures of high $T_{\mathrm{g}}$ PMPS with small concentrations of BMC. The reorientational dynamics of BMC were faster in the presence of higher $T_{\mathrm{g}}$ PMPS. While non-zero excess volume can modify the solution dynamics $[4,17]$, BMC densifies when mixed with a small concentration of PMPS [16]. 
Based on free-volume ideas $[4,18,19]$, this negative excess volume should slow the BMC motion, contrary to the experimental results. In the case of BMC/PMPS, it was concluded that the coupling model offered the only tenable hypothesis for the observed speeding up of the BMC relaxation.

By using dielectric relaxation techniques, we have extended the work on the BMC, PMPS and the corresponding mixtures, enabling the segmental dynamics and rotational mobility to be monitored over broader temperature and frequency ranges, and, in particular, above the glass transition temperatures as measured by DSC.

\section{Experimental}

The BMC, synthesized as described elsewhere [20], was filtered through $5 \mu \mathrm{m}$ paper immediately prior to use; this slows down the crystallization rate presumably by removing nucleating impurities. The filtered BMC could be quenched without crystallization, as confirmed by DSC. Without this filtering, the relaxation behavior of $\mathrm{BMC}$ and the BMC-rich mixtures in the vicinity of $T_{\mathrm{g}}$ was poorly reproducible. The poly(methylpheylsiloxanes) were prepared by an anionic ring-opening polymerization of 1,3,5-trimethyl-1,3,5-diphenylcyclotrisiloxane; the full procedure and sample characterization is described elsewhere [21]. The number average molecular weights, as determined by GPC, were 12000 and 130000 , respectively, for the polymers designated herein as PMPS-l and PMPS-h, Polydispersities in both cases equaled 1.04.

Mixtures were obtained by dropwise addition of the BMC to thin films of the polymer. After absorption of the $\mathrm{BMC}$, the mixture was annealed at least $24 \mathrm{~h}$ at $50^{\circ} \mathrm{C}$ with periodic mechanical agitation, followed by $\mathrm{RT}$ annealing for one week.

Dielectric measurements were performed in the frequency range from 20 to $10^{6} \mathrm{~Hz}$ using a Hewlett-Packard impedance analyzer. The samples were placed between two condenser plates (gold plated stainless steel electrodes), inside a cryostat in which the sample temperature was regulated via a stream of temperature-controlled nitrogen gas.

Dynamic mechanical data in the vicinity of the glass transition zone were obtained with a Bohlin
VOR rheometer using a parallel plate geometry. Sample radii and gaps were typically 6 and $2 \mathrm{~mm}$, respectively. The dynamic shear modulus was usually measured from $20 \mathrm{~Hz}$ down to as low as $1 \times 10^{-4} \mathrm{~Hz}$. the broad range of experimental frequencies obviated time-temperature superpositioning (which is usually not valid for blends [22-27] nor even for some neat polymers $[28,29])$. Prior to measurements at any given temperature, samples were maintained for a time period exceeding the anticipated relaxation time, in order to minimize errors due to physical aging, an absence of which was affirmed by the reproducibility of measurements repeated after various time periods.

\section{Results}

Fig. 1 displays representative measurements of the dynamical mechanical loss modulus in the $T_{\mathrm{g}}$ region for the two siloxane polymers and for the $\mathrm{BMC}$, while typical dielectric dispersion data are presented in Fig. 2 for the same materials at approximately $270 \mathrm{~K}$. Also shown in Figs. 1 and 2 are best fits to the Kohlrausch-Williams-Watts (KWW) stretched exponential function [30,31]

$$
E(t)=\exp \left[-\left(t / \tau^{*}\right)^{1-n}\right]
$$

using

$E^{\prime \prime}(\omega)=\omega \int_{0}^{\infty} E(t) \cos (\omega t) \mathrm{d} t$.

In none of these measurements was there any evidence of multiple glass transitions, as has been reported for a few other mixtures of small molecules with polymers [32-37]. Note that the $\tau^{*}$ in Eqs. (1) and (2) is very nearly equal to $\left(2 \pi f_{p}\right)^{-1}$, where $f_{p}$ is the frequency of the maximum in either the dynamic loss modulus or dielectric dispersion. According to the coupling model $[8,9]$, broader dispersions (larger $n$ values) for amorphous polymers are associated with more intermolecular cooperativity, while a narrow dispersion implies weaker constraints on the relaxation from nonbonded neighbors. The best-fit values for $n$ are listed in Tables 1 and 2 for the dynamical mechanical and dielectric results, respectively. The 


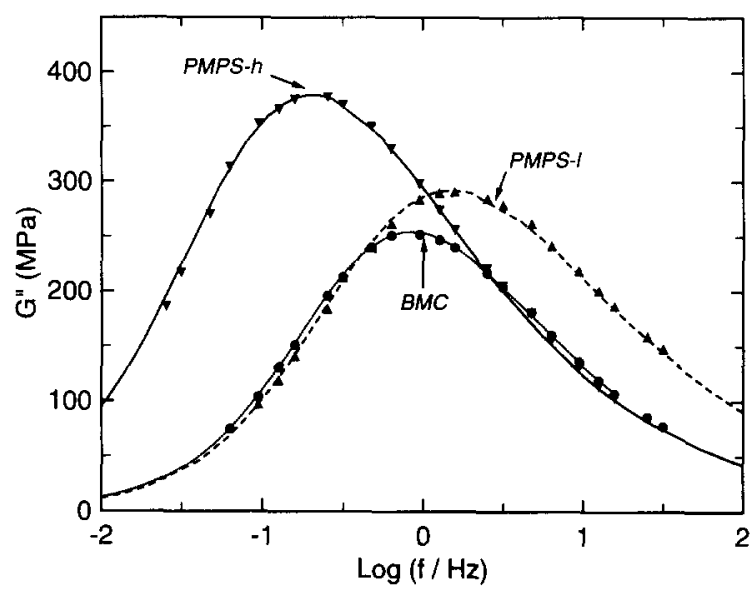

Fig. 1. Dynamic mechanical loss moduli measured for the neat material in the vicinity of their respective glass transition temperatures, along with the best-fit curve calculated using Eqs. (2) and (3). For the polymers, semi-logarithmic plotting emphasizes the contribution of segmental motion, and de-emphasizes that of the Rouse modes.

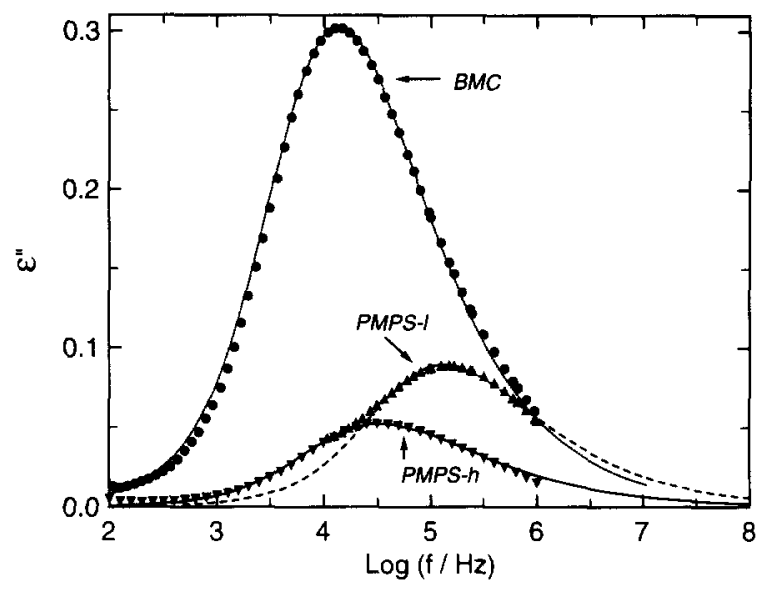

Fig. 2. Dielectric dispersion data measured for the neat PMPS polymers and the $\mathrm{BMC}$ at approximately $270 \mathrm{~K}$, along with the best-fit curve calculated using Eqs. (2) and (3).

magnitude of $n$ was only weakly dependent on temperature, varying no more than \pm 0.02 over the range of temperatures for the mechanical measurements and \pm 0.03 over the range of temperatures for the dielectric data.
Table 1

Dynamical mechanical spectroscopy results for neat liquids and mixtures $^{\mathrm{a}}$

\begin{tabular}{|c|c|c|c|c|c|}
\hline & $T_{\mathbf{g}}^{\mathbf{b}}$ & $\log A$ & $B$ & $T_{\infty}$ & $n$ \\
\hline BMC & 241.0 & -20.79 & 3556 & 173.2 & 0.44 \\
\hline PMPS-1 & 237.4 & -21.96 & 2693 & 188.6 & 0.50 \\
\hline PMPS-h & 243.2 & -15.69 & 1414 & 208.5 & 0.50 \\
\hline $90 \% \mathrm{BMC}$ & & & & & \\
\hline $\begin{array}{l}\text { in PMPS-1 } \\
90 \% \text { BMC }\end{array}$ & 240.5 & -15.16 & 1868 & 193.2 & 0.44 \\
\hline $\begin{array}{l}\text { in PMPS-h } \\
10 \% \text { BMC }\end{array}$ & 240.9 & -17.22 & 2427 & 186.0 & 0.44 \\
\hline $\begin{array}{l}\text { in PMPS-1 } \\
10 \% \text { BMC }\end{array}$ & 238.7 & -7.43 & 303 & 224.7 & 0.51 \\
\hline in PMPS-h & 242.5 & -10.47 & 667 & 219.3 & 0.48 \\
\hline
\end{tabular}

${ }^{a}$ Errors are given in the text.

${ }^{b}$ Corresponding to the temperature at which the mechanical relaxation time equals $100 \mathrm{~s}$.

Table 2

Dielectric spectroscopy results for neat liquids and mixtures ${ }^{a}$

\begin{tabular}{|c|c|c|c|c|c|}
\hline & $T_{\mathrm{g}}^{\mathrm{b}}$ & $\log A$ & $B$ & $T_{\infty}$ & $n$ \\
\hline BMC & 267.3 & -26.84 & 6920 & 135.7 & 0.44 \\
\hline PMPS-1 & 258.4 & -11.32 & 548 & 225.9 & 0.49 \\
\hline PMPS-h & 261.8 & -11.47 & 621 & 225.7 & 0.47 \\
\hline $\begin{array}{l}90 \% \text { BMC } \\
\text { in PMPS-I }\end{array}$ & 265.6 & -19.27 & 2969 & 181.2 & 0.44 \\
\hline $90 \% \mathrm{BMC}$ & & & & & \\
\hline $\begin{array}{l}\text { in PMPS-h } \\
10 \% \mathrm{BMC}\end{array}$ & 262.3 & -10.42 & 458 & 231.3 & 0.52 \\
\hline $\begin{array}{l}\text { in PMPS-1 } \\
10 \% \text { BMC }\end{array}$ & 258.1 & -12.67 & 834 & 216.4 & 0.48 \\
\hline in PMPS-h & 262.1 & -12.87 & 876 & 219.2 & 0.48 \\
\hline
\end{tabular}

${ }^{a}$ Errors are given in the text.

${ }^{b}$ Corresponding to the temperature at which the dielectric relaxation time equals $0.0001 \mathrm{~s}$.

The $n$ values obtained for BMC are consistent with, albeit slightly smaller than, those previously reported from dielectric spectroscopy measurements [38]. Although a dependence of the observed relaxation function on the experimental probe used to measure it would not be without precedent $[10,39,40]$, we believe at least part of the difference between both the mechanical and dielectric results herein and the earlier dielectric data [38] is due to 
the different manner in which the respective data, all obtained in the frequency domain, were fitted to the time domain KWW function [41]. Although the two polymers have different glass transition temperatures (and hence different at any given temperature), it has been previously demonstrated that the shape of the segmental relaxation dispersion, and hence the degree of intermolecular coupling, is not a function of molecular weight $[16,42]$.

Although the distribution of local environments engendered by concentration fluctuations should broaden both the mechanical loss modulus peaks and dielectric dispersions relative to those of the neat liquids [23,24,43-45], this effect is seen to make a minor contribution to the measured mech-

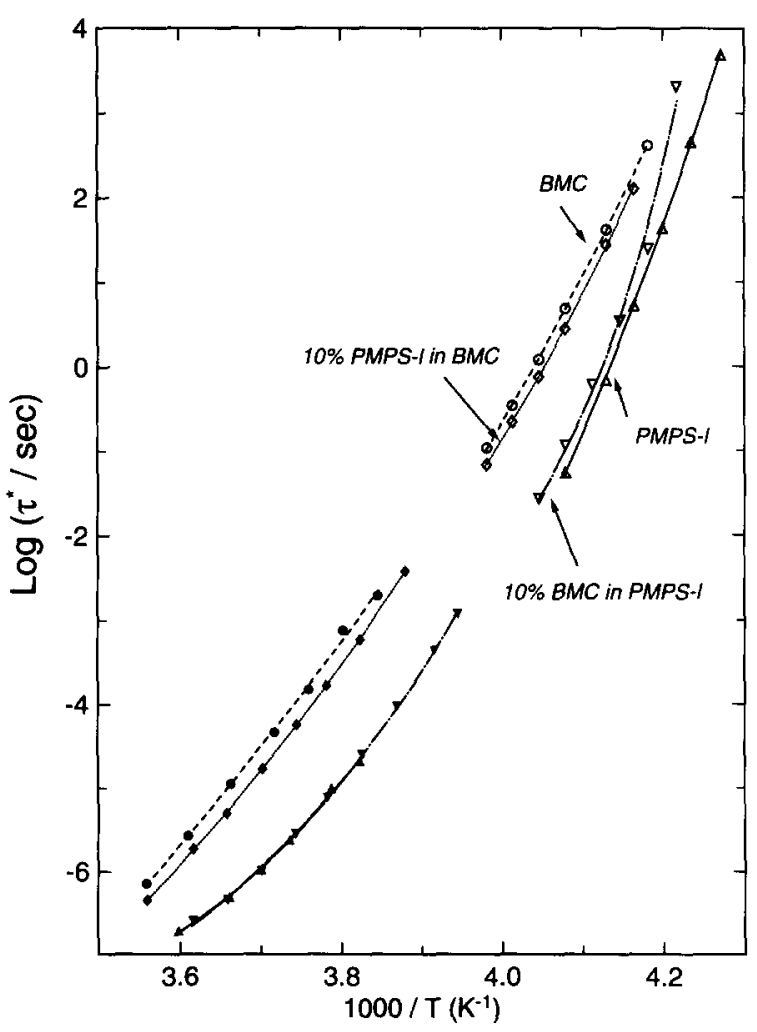

Fig. 3. The relaxation times (defined as $\left(2 \pi f_{\mathrm{p}}\right)^{-1}$, where $f_{\mathrm{p}}$ is the frequency of the maximum in the dispersion) for BMC, PMPS-1 and their mixtures. The open and filled symbols represent the relaxation times measured by dynamical mechanical and dielectric spectroscopies, respectively. In this and in Fig. 4, the solid curves represent the results of fitting Eq. (4) to the data. anical dispersions. This apparent absence of significant inhomogeneous broadening allows direct application of Eq. (2), even though in principle the viscoelastic spectra of blends will not exhibit the KWW shape [23,24]. The results (Tables 1 and 2; Ref. [16]) suggest that the components upon mixing exhibit very nearly the coupling parameter observed for the neat material. Of course, the possibility also exists that some inhomgeneous broadening is negated by a concurrent reduction in the intermolecular coupling of either component upon mixing. When measured with the dynamical mechanical technique, BMC containing $10 \%$ of either PMPS may have a slightly smaller coupling parameter, with this reduction of $n$ compensated by a modest inhomogenous broadening. However, when measured by dielectric spectroscopy, it is interesting to note that $\mathrm{BMC}$ containing $10 \%$ of PMPS- $h$ is an exception, having a broader loss dispersion that neat BMC. In general, there is no reason to expect that the shape of the relaxation function, time-temperature shift factors, and correlation times measured by the two spectroscopies be equivalent [10].

Figs. 3 and 4 displays the temperature dependence of the relaxation times measured for various mixtures of BMC with low molecular weight PMPS-1 and high molecular weight PMPS-h, respectively. Also included in the figures are the best fits of the Vogel-Fulcher equation [18] (see Tables 1 and 2)

$\tau^{*}=A \exp \left(B /\left(T-T_{\infty}\right)\right.$.

(Due to the non-uniqueness of fitting three parameters in the Vogel-Fulcher equation, the best-fit curves are shown only as a visual aid and should be interpreted as such.)

\section{Discussion}

\subsection{BMC-rich mixtures}

In Fig. 3, the addition of 10\% PMPS-1 to BMC reduces the latter's relaxation time, as expected simply from $T_{\mathrm{g}}$ considerations. Since the PMPS-h has a higher glass transition temperature than BMC, its addition might be expected to slow down 


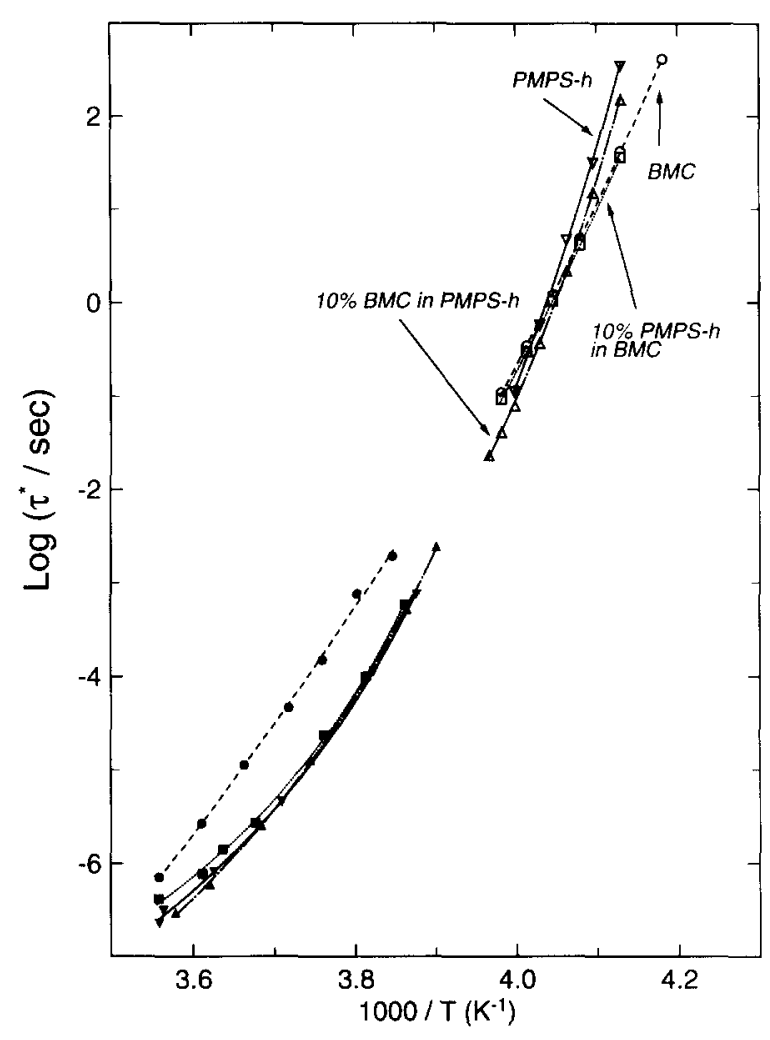

Fig. 4. The temperature dependence of the relaxation times for various mixtures of BMC with PMPS-h measured mechanically (open symbols) and dielectrically (filled). BMC plasticizes the PMPS-h relaxation, while, over the range of the measurements, the higher $T_{\mathrm{g}}$ polymer reduces the relaxation time of the lower $T_{\mathrm{g}} \mathrm{BMC}$.

BMC reorientation. On the contrary, however, at all temperatures the $\mathrm{BMC}$ relaxation time is observed to decrease upon addition of $10 \%$ PMPS-h (Figs. 4 and 5(a)).

A similar anomaly appears in PCB/PVE mixtures, where again a higher $T_{\mathrm{g}}$ polymer increases the relaxation rate of a lower $T_{\mathrm{g}}$ small molecule liquid. A hypothesis has been advanced to explain the unexpected nature of the modification of PCB's dynamics by PVE [5]. This explanation assumes that the magnitude of the local friction in the mixture is reflected in the primitive relaxation time, $\tau_{0}$ (i.e., relaxation absent intermolecular coupling), deduced via Eq. (1) from measurements on the neat liquids. Because the neat polymer is associated with stronger intermolecular coupling than is the neat $\mathrm{PCB}$, there is a reversal in the relative magnitudes of the relaxation times in going from $\tau^{*}$ to $\tau_{0}$ via Eq. (1). Of course, such a calculation of $\tau_{0}$ is only a 'first-order' treatment, which circumvents detailed consideration of how the free volume and potential field arising from the local environment influences that actual $\tau_{0}$ in the mixture.

Nevertheless, we apply Eq. (1) together with the $n$ measured for the neat liquids to obtain at least qualitative predictions of the effect that PMPS-h has on the BMC reorientational dynamics. Taking $10^{11} \mathrm{rad} / \mathrm{s} \leqslant \omega_{\mathrm{c}} \leqslant 10^{12} \mathrm{rad} / \mathrm{s}, \tau_{0}$ for $\mathrm{BMC}$ is found to be longer than the corresponding primitive relaxation time of PMPS-h, even though $\tau_{\text {BMC }}^{*}<\tau_{\text {PMPS }-\mathrm{h}}^{*}$ (see Fig. 5(a)). Thus, 10\% PMPS-h decreases the local friction, contributing to faster $\mathrm{BMC}$ relaxation. The anomalous decrease in BMC's relaxation time can be reconciled in terms of the reversal in the magnitude of the respective relaxation times upon reduction in the strength of the effect of intermolecular coupling; i.e., $\tau_{\text {BMC }}^{*}<\tau_{\text {PMPS.h }}^{*}$ but $\tau_{0, \text { BMC }}>\tau_{0, \text { PMPS }}$ (Fig. 5(a)). The fact that $\tau_{0 \mathrm{BMC}}>\tau_{0, \mathrm{PMPS}}$ means that the guest polymer molecules have higher intrinsic (on the $\tau_{0}$ level) mobility than that of the host BMC molecules, at least before cooperative constraint dynamics are considered. As a consequence, the guest molecules will mitigate the mutual dynamic constraints among the BMC molecules, leading to their faster motion (i.e., a decrease of $\tau_{\mathrm{BMC}}^{*}$ as described by Eq. (1) with a decrease in the coupling parameter).

As discussed earlier, polymer solution additivity of the volumes is not expected, and it must be recognized that a non-zero excess volume can contribute to changes in the relaxation times $[4,19]$. While densification is more common for mixtures of small molecules with polymers $[12,14,46]$, positive volume changes upon mixing are known [47].

In Fig. 6, the density measured at room temperature is shown for neat BMC and PMPS-h, as well as for the $10 \%$ polymer mixtures. The excess volume, while very small, is negative for the BMC-rich solution. This would contribute to a slowing down of the BMC motion, notwithstanding that experimentally the relaxation accelerates. Thus, the reversal in $\tau_{0}$ s discussed above (Fig. 5) must be at least the primary, if not the only, factor governing the 

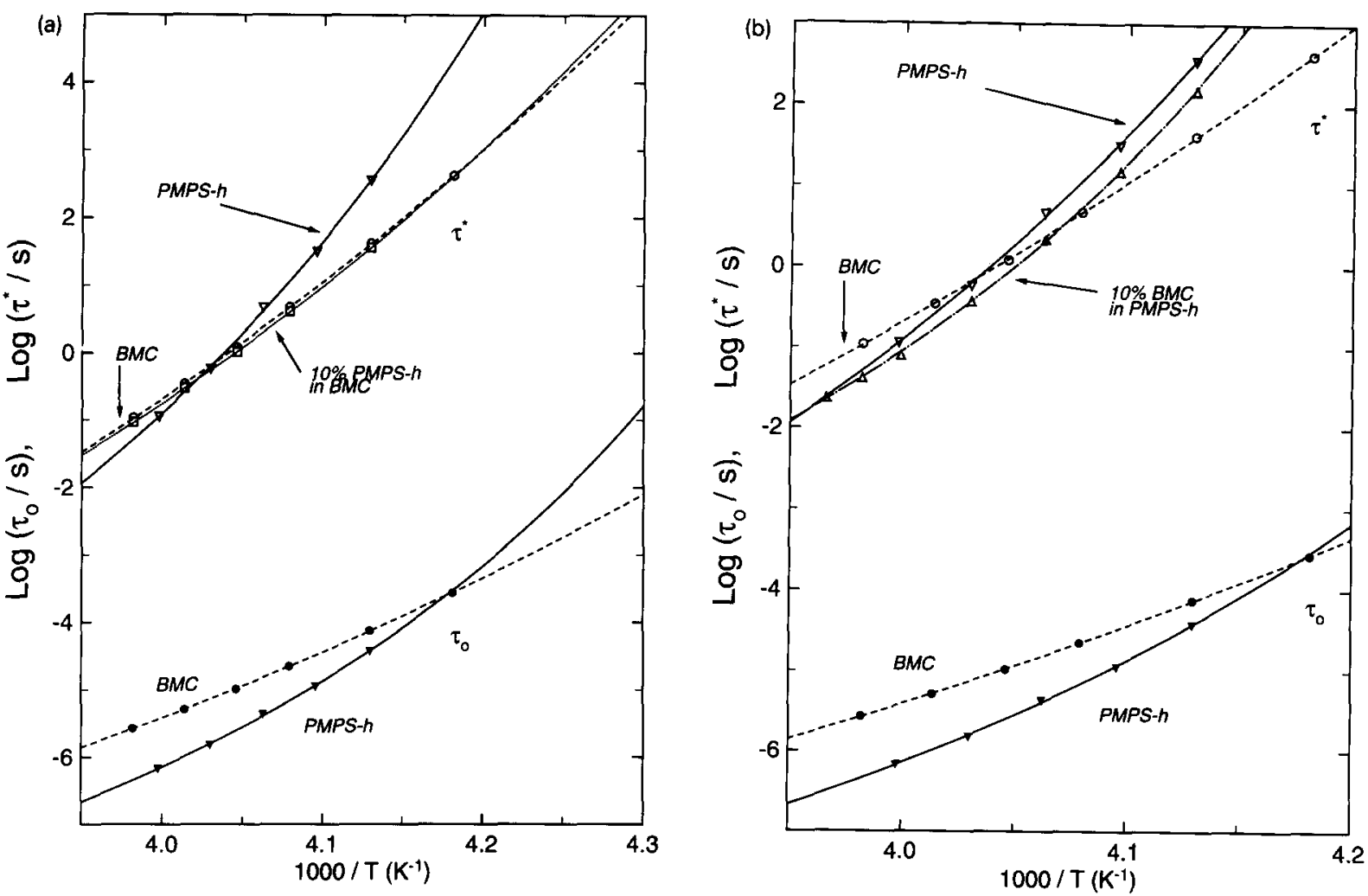

Fig. 5.(a) The relaxation times of neat BMC and PMPS-h, along with the results for a mixture containing $10 \%$ of the polymer. The lower curves in this and in Fig. 5(b) represent the values of $\tau_{0}$ calculated using Eq. (1) from the $\tau^{*}$ measured for the neat components. This reversal in $\tau_{0}$ gives rise to the anomalous decrease in relaxation time of BMC upon addition of the higher $T_{\mathrm{g}}$ PMPS-h. (b) The relaxation times of neat BMC and PMPS-h, along with the results for a mixture containing $10 \%$ of the BMC. Notwithstanding the reversal in the relative magnitudes of the relaxation times upon removal of intermolecular cooperativity effects, the absence of the predicted anti-plasticization of PMPS-h upon addition of BMC may be a result of the additional free volume arising from mixing.

modification of BMC dynamics upon addition of the PMPS-h.

Another interesting feature in Figs. 4 and 5 is the apparent inversion of the respective relaxation times for the neat material as temperature increases above the DSC measured glass transition temperature. This observation is confirmed in Fig. 4 by the reversal in the relaxation times as measured by the dielectric spectroscopy (i.e. $\tau_{\mathrm{BMC}}^{*}>\tau_{\mathrm{PMPS}-\mathrm{h}}^{*}$ ); thus, at high temperatures, the higher $T_{\mathrm{g}}$ polymer has a shorter relaxation time!

The directional changes in the relaxation times for the polymers upon addition of $10 \% \mathrm{BMC}$ show 'conventional' behavior for the dielectric data (Figs. 3 and 4). That is, the mixture transition tem- peratures are intermediate to those of the pure components. However, the apparent acceleration of the $\mathrm{BMC}$ rotational mobility in the $10 \%$ polymer mixtures is incompatible with the observed negative excess volume for the BMC-rich solution, but is consistent with Eq. (1) (i.e., $\tau_{\mathrm{BMC}}^{*}>\tau_{\mathrm{PMPS}-\mathrm{h}}^{*}$ and $\left.\tau_{0, \text { BMC }}>\tau_{0, \text { PMPS-h }}\right)$. We do note that the BMC-rich mixture exhibits a relaxation time almost equivalent to the neat PMPS-h (Fig. 4).

Further, the reversal in the relaxation time of the $10 \%$ PMPS-h mixture as the temperature is decreased far below the DSC measured glass transition temperature is consistent with the reversal predicted in the $\tau_{0}$ values for the neat material (Fig. 5(a)). Again, since $\tau_{0}$ is only a 'first order' treatment 


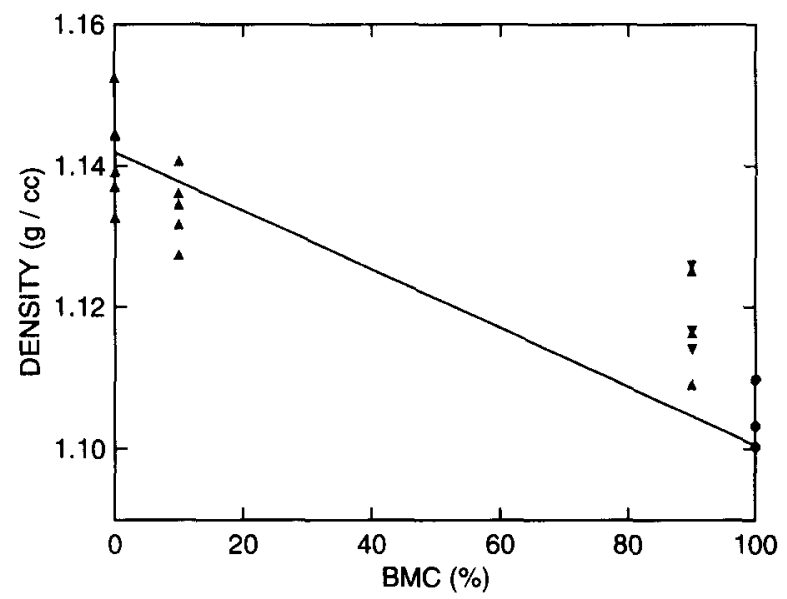

Fig. 6. The density measured for the mixtures at room temperature (the symbols correspond to those in Fig. 1). The solid line represents linear interpolation between the pure liquid densities. Note that addition of PMPS densifies the BMC, incongruent with the observed decrease of the latter's relaxation time. The polymer-rich mixture may be associated with a slight positive excess volume, but the result is not greater than the scatter in the data.

of the data, the slight deviation in temperature for the predicted crossing point of $\tau_{0}$ values and the observed reversal in relaxation times. $\tau^{*}$, is acceptable (Fig. 5).

\subsection{PMPS-rich mixtures}

The directional changes in the relaxation times of the polymers upon addition of $10 \% \mathrm{BMC}$ are not surprising; BMC plasticizes the higher $T_{\mathrm{g}}$ PMPS-h (Figs. 4 and 5(b)) and anti-plasticizes the lower $T_{\mathrm{g}}$ PMPS-1 (Fig. 3). This is 'conventional' behavior in that it is consistent with the component glass transition temperatures; however, it begs the question why the reversal seen in the BMC-rich mixture ( $\tau_{\text {BMC }}^{*}<\tau_{\text {PMPS-h }}^{*}$ but $\tau_{0, \text { BMC }}>\tau_{0, \text { PMPS-h }}$ ) is not observed for PMPS-h containing 10\% BMC. The prediction from Eq. (1) of longer $\tau_{0}$ for BMC than for PMPS-h suggests, upon addition of BMC, an enhancement of intermolecular coupling of the PMPS, with concomitant increase in $\tau_{\text {PMPS-h}}^{*}$.

It follows that $10 \%$ BMC should slow down segmental relaxation of PMPS-h; however, such anomalous anti-plasticization of the PMPS- $h$ is not observed in Fig. 5(b). The data in Fig. 6 hint that the polymer-rich mixture may be associated with a positive excess volume. Although the result is not significantly greater than the scatter in the measurements, it is tempting to infer that the absence of the predicted anti-plasticization of PMPS$\mathrm{h}$ upon addition of BMC (since from Eq. (1), $\tau_{0, \mathrm{BMC}}$ $\left.>\tau_{0, \text { PMPS-h }}\right)$ is due to the additional free volume arising from mixing. In other words, the anti-plasticization effect predicted from the coupling model may be obscured by the plasticization effect caused by a positive excess volume.

This hypothesis can be tested by considering similar experimental measurements using PMPS samples with lower molecular weights and correspondingly lower $T_{\mathrm{g}} \mathrm{s}$. These samples will have shorter $\tau_{0} s$ than that of PMPS-h as illustrated in Fig. 7 for PMPS-1 and for a sample of PMPS (referred to as PMPS-s) that has the same $T_{\mathrm{g}}$ as that of BMC [15]. The $\tau^{*} s$ and the calculated $\tau_{0} s$ of the three PMPS samples and BMC are shown in Fig. 7. It is seen that the ratio $\tau_{0, \mathrm{BMC}} / \tau_{0, \mathrm{PMPS}}$ increases significantly when going from PMPS-h to PMPS-s to PMPS-1. We can conclude from the trend of this ratio that the predicted anti-plasticization effect will be enhanced in PMPS-s and PMPS-1 when compared to PMPS- $h$. This suggests that in PMPS-s-rich or PMPS-1-rich mixtures, the enhanced anti-plasticization effect may overcome the plasticization coming from the positive excess volume.

Evidence of this can in fact be found in the tracer diffusion measurements of Lohfink and Sillescu on mixtures of PMPS-s and BMC [15]. They found that addition of BMC to PMPS substantially reduced the diffusion constant of a probe molecule having the same size as the BMC [15]; this is in direct contradiction to the consequences of an increase in volume upon mixing. The anti-plasticization seen for 10\% BMC in PMPS-1 (Fig. 3), notwithstanding the positive excess volume of the mixture, provides additional support for an interpretation based on the second relation of the coupling model (Eq. (1)). We also note in passing that the probe diffusion measurements of Lohfink and Sillescu [15] in BMC-rich mixtures have also found an increase of mobility with addition of PMPS-s 


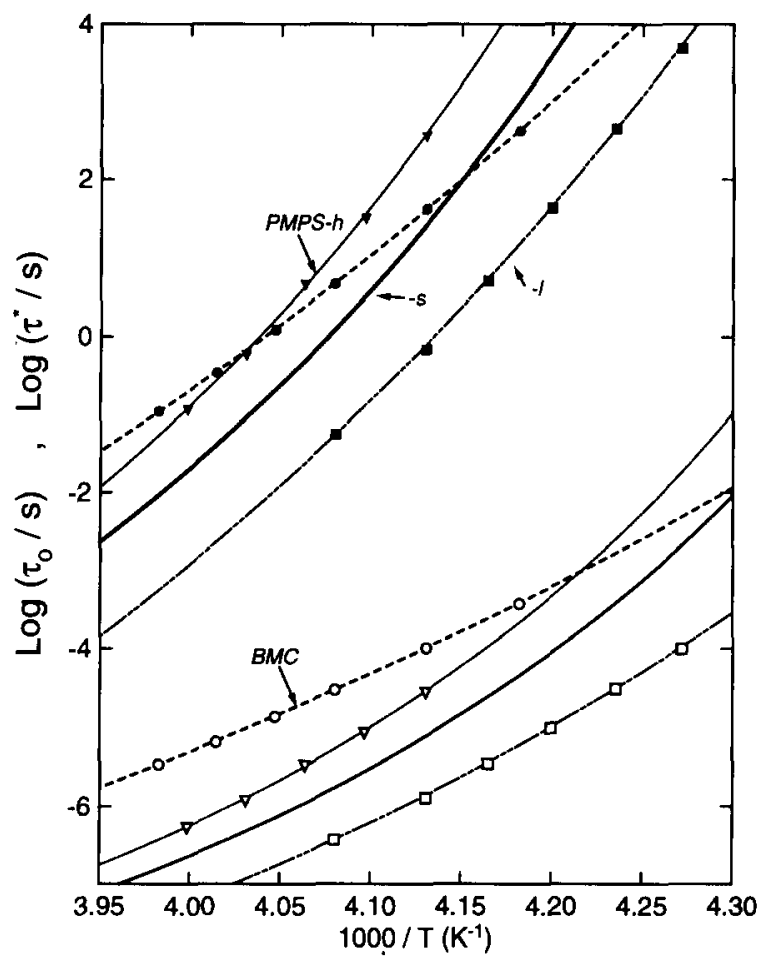

Fig. 7. The $\tau^{*}$ s for three polymers with different molecular weights, PMPS-h ( $\mathbf{\nabla})$ PMPS-s (- - ) and PMPS-1 ( $\boldsymbol{\square}^{-}$), and BMC ( - - - $\bullet$ ). The corresponding non-cooperative relaxation times, $\tau_{0}$, for these materials are indicated by the corresponding open symbols and the thinner continuous curve for PMPS-s.

analogous to our mechanical relaxation data for BMC-rich mixtures of PMPS-h.

\section{Conclusions}

For the BMC-rich mixtures we observe an anomaly - the addition of higher $T_{\mathrm{g}}$ PMPS-h reduces the BMC's relaxation time. This result is difficult to explain given the PMPS-h has a higher $T_{\mathrm{g}}$ than $\mathrm{BMC}$, and that the excess volume in BMC-rich PMPS solutions is negative (Fig. 6). This anomaly can be rationalized from the coupling model (Eq. (1)). The analysis shows that the monomeric friction coefficient, reflected in the value of $\tau_{0}$, is smaller for PMPS-h than for BMC, notwithstanding the polymer's higher $T_{\mathrm{g}}$. The value of $T_{\mathrm{g}}$ measured for a neat liquid is governed by in part intermolecular coupling. Intermolecular coupling is stronger in PMPS-h $(n=0.50)$ than in BMC ( $n=0.44)$; thus, $\tau_{\text {PMPS-h }}^{*}$ is longer even though $\tau_{0, \text { PMPS-h }}<\tau_{0, \mathrm{BMC}}$. The results herein from mechanical and dielectric spectroscopies are consistent with earlier probe diffusion experiments on mixtures PMPS and BMC having equal glass transition temperatures [15].

In PMPS-h-rich mixtures, we observe a positive excess volume which will speed up the PMPS-h segmental motion. The coupling model, limited to a comparison of $\tau_{0, \mathrm{BMC}}$ and $\tau_{0, \mathrm{PMPS}-\mathrm{h}}$, predicts slowing of PMPS segmental relaxation upon addition of BMC. It is likely that in the PMPS-h mixtures, the change in volume upon mixing dominates, whereby the coupling model prediction fails. This situation is expected to be reversed in polymer-rich mixtures using lower molecular weight PMPS; the anti-plasticization effect (Eq. (1)) will overcome the plasticization coming from the positive excess volume. This expectation has been realized in the probe diffusion results discussed earlier [15], and verified with data presented herein. A principal finding is that the primitive relaxation time, $\tau_{0}$, and the related monomeric friction coefficient are smaller for the polymer than for BMC, in spite of the relative values of $\tau^{*}$ and $T_{\mathrm{g}}$ measured for the neat liquids. Hence, addition of BMC slows down probe diffusion, and addition of PMPS- $h$ increases the relaxation rate of $\mathrm{BMC}$.

Note that only through the study of mixtures comprised of components of nearly equal $T_{\mathrm{g}}$ can the anomalies observed herein and elsewhere $[4,11,15,16]$ be observed. In the more usual situation, a large difference in component glass transition temperatures overwhelms the more subtle effects arising from Eq. (1) or excess mixing volumes; consequently, much interesting physics is undetected.

The work at NRL was supported by the Office of Naval Research (K.L.N.) in part under contract N0001493WX24011. P.G.S. expresses his gratitude for a National Research Council-Naval Research Laboratory post-doctoral fellowship. The authors gratefully acknowledge Professor G. Meier for supplying the BMC and PMPS. 


\section{References}

[1] T.P. Lodge, J. Phys. Chem. 97 (1993) 1480.

[2] J.L. Schrag et al., J. Non-Cryst. Solids 131-133 (1991) 537.

[3] D.J. Gisser and M.D. Ediger, Macromolecules 25 (1992) 1284.

[4] H. Fujita, Macromolecules 26 (1993) 643.

[5] A. Rizos and K.L. Ngai, Phys. Rev. B46 (1992) 8126.

[6] P.G. Santangelo, K.L. Ngai and C.M Roland, Macromolecules, in press.

[7] K.L. Ngai, R.W. Rendell , A.K. Rajagopal and S. Teitler, Ann. NY Acad. Sci. 484 (1986) 150.

[8] K.L. Ngai and R.W. Rendell, J. Non-Cryst. Solids 131-133 (1991) 942.

[9] C.M. Roland and K.L. Ngai, Macromolecules 24 (1991) 5315.

[10] J. Colmenero, A. Alegria, P.G. Santangelo, K.L. Ngai and C.M. Roland, Macromolecules 27 (1994) 407.

[11] C.M. Roland, Macromolecules, in press.

[12] D. Patterson, Polym. Eng. Sci. 22 (1982) 64.

[13] G.N. Lewis, M. Randal, K.S. Pitzer and L. Brewer, Thermodynamics (McGraw-Hill, New York, 1961) ch. 21.

[14] Y. Maeda and D.R. Paul, J. Polym. Sci. Polym. Phys. 25 (1987) 1005

[15] M. Lohfink and H. Sillescu, in: Proc. 1st Tohwa Univ. Int. Symp., ed. K. Kawasaki, T. Kawakatsu and M. Tokuyama (American Institute of Physics, New York, 1992).

[16] P.G. Santangelo, C.M. Roland, K.L. Ngai and G. Meier, Macromolecules 26 (1994) 6164.

[17] H. Fujita, M. Wolf and J.H. Wendorff, Polymer 30 (1989) 1524.

[18] J.D. Ferry, Viscoelastic Properties of Polymers (Wiley, New York, 1980).

[19] H. Fujita, Fortschr. Hochpolym. Forsch 3 (1961) 1.

[20] B. Gerharz, G. Meier and E.W. Fischer, J. Chem. Phys. 92 (1990) 7110

[21] B. Momper, T. Wagner, U. Maschke, M. Ballanff and E.W. Fischer, Polym. Common. 31 (1990) 286.

[22] J.B. Miller, K.J. McGrath, C.M. Roland, C.A. Trask and A.N. Garroway, Macromolecules 23 (1990) 4543.

[23] C.M. Roland and K.L. Ngai, Macromolecules 24 (1991) 2261.

[24] C.M. Roland and K.L. Ngai, J. Rheol. 36 (1992) 1691.

[25] R.J. Composto, E.J. Kramer and D.M. White, Macromolecules 21 (1988) 2580.

[26] R.J. Colby, Polymer 30 (1989) 1275.

[27] C.M. Roland, K.L. Ngai, J.M. O'Reilly and J.S. Sedita, Macromolecules 25 (1992) 3906.

[28] D.J. Plazek, J. Phys. Chem. 69 (1965) 3480.

[29] K.L. Ngai and D.J. Plazek, J. Polym. Sci. Polym. Phys. Ed. 24 (1986) 619.
[30] R. Kohlrausch, Pogg. Ann. Phys. 12 (1847) 393.

[31] G. Williams and D.C. Watts, Trans. Faraday Soc. 66, (1970) 80.

[32] R.S. Adler and K.F. Freed, Macromolecules 11 (1978) 1058.

[33] E.D. von Meerwell, S. Amelar, M.A. Smeltzly and T.P. Lodge, Macromolecules 22 (1989) 295.

[34] Y. Liu, A.K. Roy. A.A. Jones, P.T. Inglefield and P. Ogden, Macromolecules 23 (1990) 968.

[35] K. Adachi, M. Hattori and Y. Ishida, J. Polym. Sci. Polym. Phys. Ed. 15 (1977) 693.

[36] R.P. Kambour, J.D. Carbeck and W.L. Nachlis, J. NonCryst. Solids 131-133 (1991) 563.

[37] D.J. Plazek, C. Seoul and C.A. Bero, J. Non-Cryst. Solids 131-133 (1991) 570.

[38] G. Meier, B. Gerharz and D. Boese, J. Non-Cryst. Solids $131-133$ (1991) 144.

[39] N.G. McCrum, B.E. Read and G. Williams, Anelastic and Dielectric Effects in Polymer Solids (Wiley, London, 1967).

[40] K.L. Ngai, S. Mashimo and G. Fytas, Macromolecules 21 (1988) 3030.

[41] The dielectric spectroscopy determinations of $n$ in Ref. [38] were carried out by fitting the frequency dependence of the dielectric loss to the Havriliak-Negami form, and then transforming this analytical representation of the data into the time domain. The latter were then fit directly to the KWW function (Eq. (2)). This method weights the high and low frequency tails of the dispersion to a greater extent than direct fitting of the frequency data using Eq. (3). For the previously reported dielectric loss data of BMC, the transform of the Havriliak-Negami fit yields larger values of the coupling parameter $(n=0.49 \pm 0.03$ [38]) than the $n$ we obtain by direct fitting of the same data in the frequency domain; the latter emphasizes more the peak of the dispersion and yields $n=0.45$. This value is close to the coupling parameter determined herein from both mechanical and dielectric measurements on BMC.

[42] C.M. Roland and K.L. Ngai, Macromolecules 25 (1992) 5765.

[43] M.S. Shears and G. Williams, J. Chem. Soc. Faraday Trans. 269 (1973) 608.

[44] R.E. Wetton, W.J. MacKnight, J.R. Fried and F.E. Karasz, Macromolecules 11 (1978) 158.

[45] C.A. Trask and C.M. Roland, Macromolecules 22 (1989) 256.

[46] D. Patterson, Macromolecules 2 (1969) 672.

[47] P.J. Flory, Selected Works of Paul J. Flory, ed. L. Mandelkern, J.E. Mark, U.W. Suter and D.Y. Yoon, Vol. 1 (Stanford Press, Stanford, CA, 1985). 